
\title{
$\angle$ Research Square \\ Persistence of Pregabalin Treatment in Taiwan: a Nation-Wide Population-Based Study
}

\section{Yen-Feng Wang}

Taipei Veterans General Hospital

\section{Yung-Tai Chen}

Taipei City Hospital Heping Branch

\section{Ching-Wen Tsai}

Taipei Medical University

\section{Yu-Chun Yen}

Taipei Medical University

\section{Yi-Chun Chen}

Taipei Veterans General Hospital

\section{Ben-Chang Hsia}

Taipei Medical University

Shuu-Jiun Wang ( $\sim$ sjwang@vghtpe.gov.tw )

https://orcid.org/0000-0001-5179-5358

\section{Research article}

Keywords: pregabalin, pain, persistence

Posted Date: March 26th, 2020

DOI: https://doi.org/10.21203/rs.3.rs-18478/v1

License: (a) (i) This work is licensed under a Creative Commons Attribution 4.0 International License. Read Full License

Version of Record: A version of this preprint was published at The Journal of Headache and Pain on May 19th, 2020. See the published version at https://doi.org/10.1186/s10194-020-01123-4. 


\section{Abstract}

Background Pregabalin is approved for the treatment of neuropathic pain, fibromyalgia, and seizure disorders, although the pivotal trials were mostly carried out in Europe or North America. The prescribing patterns among different indications in Asia have rarely been explored.

Methods This was a population-based retrospective cohort study based on the National Health Insurance Research Database in Taiwan. Prescriptions of pregabalin were identified, and data regarding demographics, indications, co-existing diagnoses, and concomitant medications were extracted. Pregabalin users were followed for at least one year, and factors associated with persistence at one year were determined by using multivariate logistic regression analysis.

Results Between June 2012 and December 2016, 114,437 pregabalin users (mean age 60.7 \pm 15.4 years, $57.8 \%$ female) were identified. The indications included post-herpetic neuralgia (PHN) (30.5\%), musculoskeletal diseases other than fibromyalgia (21.2\%), fibromyalgia (18.4\%), diabetic peripheral neuropathic pain (DPNP) (11.7\%) and epilepsy (2.9\%). Overall, $62.5 \%$ and $6.4 \%$ of patients achieved a maximum dose of $\geq 150$ and $\geq 300$ $\mathrm{mg} /$ day, respectively. The median duration of persistent pregabalin use was 28 days (interquartile range 14-118 days). The one-year persistence rate was $12.1 \%$, and the indications associated with the highest and lowest persistence rates were epilepsy (42.4\%) and PHN (6.1\%), respectively. Male gender (odds ratio [OR] 1.14, 95\% confidence interval [Cl] 1.09-1.18), older age (OR 1.01 per year, 95\% $\mathrm{Cl} 1.01-1.01$ ), indications other than $\mathrm{PNH}$, especially epilepsy (OR 8.04, 95\% Cl 7.33-8.81, PHN as reference), and a higher initial dose (OR 1.12 per 75 mg, $95 \% \mathrm{Cl}=1.10-1.15)$ were associated with persistence at one year, whereas the initial concomitant use of antiviral agents decreased the likelihood (OR 0.41, 95\% Cl 0.35-0.47).

Conclusions Pregabalin prescriptions for pain disorders were limited to short-term use, which is consistent around the world. However, the average prescribed dose in Taiwan was lower than those in Western countries, and was typically below the recommended ranges. Potential causes included off-label prescriptions for pain in acute herpes zoster, rather than $\mathrm{PNH}$, as well as intolerance to the side effects. Keywords pregabalin, pain, persistence

\section{Introduction}

Pregabalin is a new-generation antiepileptic drug targeting at the calcium channel $a 2-\delta$ subunit, and is of proven efficacy in treatment of peripheral neuropathic pain (NeP), such as post-herpetic neuralgia (PHN), and diabetic peripheral neuropathic pain (DPNP), as well as central neuropathic pain resulting from spinal cord injury. Besides, pregabalin had also been shown to be effective in the treatment of fibromyalgia and as an adjunct therapy for partial-onset seizures. Therefore, pregabalin was approved for the above indications (1). In fact, it is recommended as the fist-line agent in the treatment of NeP, particularly PHN and DPNP (2-6). Besides, with regard to the pharmacological treatment in fibromyalgia, pregabalin is one of the drugs of choice according to the evidence available (7-9). In addition, pregabalin had level A evidence for its efficacy against treatmentresistant adult focal epilepsy according to the guidelines of the American Academy of Neurology (10). It was launched in Taiwan in 2009, and became reimbursed for PHN/epilepsy, fibromyalgia, and DPNP by the Taiwan National Health Insurance (NHI) in February 2012, February 2013, and January 2016, respectively. However, the pattern of clinical practice regarding its use in Taiwan had not been explored. 
Pregabalin is generally considered well tolerated, and the commonly reported side effects include dizziness, somnolence, peripheral edema, and body weight gain (11). Although side effects are not uncommon, the dropout rates of pregabalin in clinical trials of neuropathic pain and seizure disorders were comparable to other newergeneration anticonvulsants $(10,12)$. On the other hand, although a large proportion of patients experienced adverse effects in clinical trials of fibromyalgia, the occurrence of serious adverse events was rare. The discontinuation rates of pregabalin treatment group were, in fact, comparable to those of the placebo group (13, 14). However, most clinical trials were of relatively short durations and involved a highly selected population, whether the results could reflect what happens in the real-world practice is not without doubt. More importantly, the vast majority of the pivotal trials were carried out in Europe or North America, in which patients of Asian descent were under-presented (11). Therefore, the present study aimed at evaluating the long-term treatment persistence of pregabalin in the general population by using a nation-wide claims-based dataset in Taiwan.

\section{Methods}

\section{Data source}

This population-based retrospective cohort study was conducted utilizing the NHI Research Database (NHIRD) in Taiwan. The NHI is a social health insurance program integrating all public insurance systems into a single-payer system launched in 1995, and covers nearly $99 \%$ of the population for comprehensive health care in Taiwan. NHIRD is maintained by the Taiwan Health and Welfare Data Science Center. These databases provide comprehensive utilization and enrollment information including demographic data, outpatient visit records, hospitalization records, and drug prescription registry for all patients. Diseases were coded according to the 2001 International Classification of Diseases, the Ninth Revision, Clinical Modification (ICD-9-CM) before 2016, and the Tenth Revision (ICD-10) after 2016. The diagnosis codes for the diseases of interest were listed in Supplementary Table.

\section{Study sample}

In this nation-wide population-based retrospective cohort study, all pregabalin prescriptions between February 2012 and December 2016 were identified. The index date was defined as the date of the first pregabalin prescription. Data regarding demographics (including age, sex, monthly income, and residence), diagnoses, and drug prescriptions were extracted, based on which Charlson Comorbidity Index (CCl) scores were calculated. Concomitant use of other medications that could be a confounding factor in the analysis of pregabalin use was also taken into account. Patients with missing data or with follow-up periods less than 12 months were excluded. All subjects were followed until death or the end of the study (Dec 31, 2017).

\section{Pregabalin prescriptions}

For each pregabalin prescription, we collected the information on the drug type, quantity, dispensing date, and days of drug supply. The period of pregabalin use was defined as the duration between the first day of drug prescription and the end of the last-dispensed drug supply. When a patient filled a subsequent prescription within 90 days after the end of the previous supply, he or she was considered to have received continuous therapy. An interval of $>90$ days between prescription refills was defined as discontinuation of medication.

\section{Statistical analysis}


Descriptive statistics (means, standard deviations [SDs], and frequencies) were used to characterize the study population at baseline. The Charlson Comorbidity Index score was used to determine the health condition of individual patients. During the follow-up period, we calculated the overall numbers and percentages of persistent pregabalin use. Univariate and multivariate, stepwise, logistic regressions were used to identify the factors in association with continued use of pregabalin at one-year follow-up period. Factors with a $p$-value $<0.1$ in the univariate analyses were entered into the multivariate analyses, and the odds ratio (OR) (95\% confidence interval, $\mathrm{Cl}$ ) were determined. All statistical analyses were conducted with the SAS 9.4 (SAS Institute. Inc., Cary, NC). A pvalue $<0.05$ was considered to be statistically significant.

\section{Results}

\section{Characteristics of the study population (Table 1 )}


Table 1

Baseline characteristics of patients using pregabalin.

\begin{tabular}{|c|c|}
\hline & $\begin{array}{l}\text { Number } \\
\text { (percentage) }\end{array}$ \\
\hline No. of patients & 114437 \\
\hline Male & $48316(42.2)$ \\
\hline Age, mean (SD), year & $60.66(15.4)$ \\
\hline \multicolumn{2}{|l|}{ Monthly income NTS } \\
\hline NT 0-21,009 & $36963(32.3)$ \\
\hline NT $210,10-36,825$ & $52546(45.9)$ \\
\hline$>36,825$ & $24928(21.8)$ \\
\hline \multicolumn{2}{|l|}{ Region of Taiwan } \\
\hline Northern region & $49791(43.5)$ \\
\hline Central region & 33899 (29.6) \\
\hline Southern region & $27122(23.7)$ \\
\hline Eastern region & 3625 (3.2) \\
\hline \multicolumn{2}{|l|}{ Charlson Comorbidity Index score } \\
\hline 0 & $39922(34.9)$ \\
\hline 1 & $26902(23.5)$ \\
\hline $2-4$ & $34764(30.4)$ \\
\hline$>4$ & $12849(11.2)$ \\
\hline \multicolumn{2}{|l|}{ Specialties } \\
\hline Neurology & $53910(47.1)$ \\
\hline Anesthesiology & $5541(4.8)$ \\
\hline Physiatry & $3151(2.8)$ \\
\hline Psychiatry & $430(0.4)$ \\
\hline Rheumatology & $9201(8.0)$ \\
\hline Others & $42204(36.9)$ \\
\hline \multicolumn{2}{|l|}{ Indications } \\
\hline Herpes zoster & 34919 (30.5) \\
\hline Diabetic peripheral neuropathic pain & $13369(11.7)$ \\
\hline
\end{tabular}




\begin{tabular}{|l|l|}
\hline & $\begin{array}{l}\text { Number } \\
\text { (percentage) }\end{array}$ \\
\hline Fibromyalgia & $21051(18.4)$ \\
\hline Epilepsy & $3301(2.9)$ \\
\hline Other musculoskeletal diseases & $24227(21.2)$ \\
\hline Others & $17570(15.4)$ \\
\hline Concomitant medications & \\
\hline Gabapentin & $14114(12.3)$ \\
\hline Duloxetine & $4410(3.9)$ \\
\hline NSAlDs & $68679(60)$ \\
\hline Acetaminophen & $45834(40.1)$ \\
\hline Tramadol & $5011(4.4)$ \\
\hline Acetaminophen/tramadol & $31777(27.8)$ \\
\hline Lidocaine medicated plaster & $1114(1.0)$ \\
\hline Anti-viral agents & $6625(5.8)$ \\
\hline Daily dosage & \\
\hline$<75$ mg & $1162(1.0)$ \\
\hline $75-149$ mg & $57106(49.9)$ \\
\hline $150-224$ mg & $50925(44.5)$ \\
\hline$>=225$ mg & $5244(4.6)$ \\
\hline * Including valaciclovir,ganciclovir, famciclovir, and acyclovir. \\
\hline
\end{tabular}

During the study period, 114,437 pregabalin users (mean age was $60.7 \pm 15.4$ years, $57.8 \%$ female) were identified. It was mostly commonly prescribed by neurologists $(47.1 \%)$. The major indications were pain control for PNH (30.5\%), musculoskeletal diseases other than fibromyalgia (21.2\%), fibromyalgia (18.4\%), DPNP (11.7\%), and epilepsy (2.9\%). The mean initial daily dose of pregabalin was $116.8 \pm 58.2 \mathrm{mg}$ per day, and $94.4 \%$ were between $75 \mathrm{mg}$ and $225 \mathrm{mg}$ per day. Commonly used concomitant medications included non-steroidal antiinflammatory drugs (60.0\%), acetaminophen (40.1\%), acetaminophen/tramadol $(27.8 \%)$, and others. Besides, anti-viral agents were given along with the initial prescription of pregabalin in $5.8 \%$.

\section{Pregabalin prescriptions during the follow-up period}

The mean duration of persistent pregabalin use was $149.5 \pm 299.8$ days (median 28 , interquartile range [IQR] 14118) (Table 2). By 90 days after the index date, 33,570 users (29.3\%) were still using pregabalin, and pregabalin use persisted for more than one year in 13,885 (12.1\%). Among the indications for pregabalin use, epilepsy (42.4\%) and DPNP (21.7\%) had relatively higher persistence rates by one year, with the mean durations of 515.6 
\pm 603.5 and $222.0 \pm 330.1$ days, respectively. On the other hand, the one-year persistence rates were lower for fibromyalgia (11.7\%) and PHN (6.1\%), and the mean durations were $150.8 \pm 302.8$ and $89.4 \pm 224.1$ days, respectively. During follow-up, the mean prescribed dose was $140.7 \pm 92.8 \mathrm{mg}$, with the highest and lowest doses for each prescription being epilepsy (200.9 $\pm 145.4 \mathrm{mg} /$ day) and DPNP $(131.2 \pm 93.0 \mathrm{mg} /$ day), respectively (Table 3). The maximum dose prescribed was $<150 \mathrm{mg} /$ day in $37.5 \%$ of patients, and only $6.4 \%$ reached a dose of $\geq 300 \mathrm{mg} /$ day. However, the maximum dose exceeded $300 \mathrm{mg} /$ day in $24.9 \%$ of the prescriptions for epilepsy. 
Table 2

Persistence of pregabalin during follow-up.

\begin{tabular}{|c|c|c|c|c|c|c|c|}
\hline & \multicolumn{3}{|c|}{$\begin{array}{l}\text { Duration of } \\
\text { persistence (days) }\end{array}$} & \multicolumn{4}{|c|}{ No. of patients with persistent pregabalin use } \\
\hline & $\begin{array}{l}\text { No. of } \\
\text { patients }\end{array}$ & Mean(SD) & $\begin{array}{l}\text { Median } \\
\text { (IQR) }\end{array}$ & >=90days & $>=180$ days & $>=1$ year & $>=2$ years \\
\hline All cohort & 114,437 & $\begin{array}{l}149.5 \\
(299.8)\end{array}$ & $\begin{array}{l}28 \\
(14- \\
118)\end{array}$ & $\begin{array}{l}33570 \\
(29.3)\end{array}$ & $\begin{array}{l}22201 \\
(19.4)\end{array}$ & $\begin{array}{l}13885 \\
(12.1)\end{array}$ & $\begin{array}{l}33570 \\
(29.3)\end{array}$ \\
\hline \multicolumn{8}{|l|}{ Specialties } \\
\hline Neurology & 53,910 & $\begin{array}{l}179.3 \\
(330.4)\end{array}$ & $\begin{array}{l}41 \\
(14- \\
161)\end{array}$ & $\begin{array}{l}18423 \\
(34.2)\end{array}$ & $\begin{array}{l}12623 \\
(23.4)\end{array}$ & $\begin{array}{l}8165 \\
(15.1)\end{array}$ & $\begin{array}{l}18423 \\
(34.2)\end{array}$ \\
\hline Anesthesiology & 5541 & $\begin{array}{l}189.8 \\
(358)\end{array}$ & $\begin{array}{l}42 \\
(14- \\
166)\end{array}$ & $\begin{array}{l}1928 \\
(34.8)\end{array}$ & $\begin{array}{l}1302 \\
(23.5)\end{array}$ & $829(15)$ & $\begin{array}{l}1928 \\
(34.8)\end{array}$ \\
\hline Physiatry & 3,151 & $\begin{array}{l}143.4 \\
(301.1)\end{array}$ & $\begin{array}{l}28 \\
(14- \\
99)\end{array}$ & $\begin{array}{l}845 \\
(26.8)\end{array}$ & $566(18)$ & $\begin{array}{l}364 \\
(11.6)\end{array}$ & $\begin{array}{l}845 \\
(26.8)\end{array}$ \\
\hline Psychiatry & 430 & $\begin{array}{l}222.8 \\
(383.9)\end{array}$ & $\begin{array}{l}56 \\
(21- \\
209)\end{array}$ & $\begin{array}{l}162 \\
(37.7)\end{array}$ & 117 (27.2) & $\begin{array}{l}80 \\
(18.6)\end{array}$ & $\begin{array}{l}162 \\
(37.7)\end{array}$ \\
\hline Rheumatology & 9,201 & $\begin{array}{l}206.6 \\
(366.3)\end{array}$ & $\begin{array}{l}47 \\
(21- \\
191)\end{array}$ & $\begin{array}{l}3444 \\
(37.4)\end{array}$ & $2388(26)$ & $\begin{array}{l}1558 \\
(16.9)\end{array}$ & $\begin{array}{l}3444 \\
(37.4)\end{array}$ \\
\hline Others & 42,204 & $\begin{array}{l}93.3 \\
(211.3)\end{array}$ & $\begin{array}{l}22(7- \\
69)\end{array}$ & $\begin{array}{l}8768 \\
(20.8)\end{array}$ & $\begin{array}{l}5205 \\
(12.3)\end{array}$ & $\begin{array}{l}2889 \\
(6.8)\end{array}$ & $\begin{array}{l}8768 \\
(20.8)\end{array}$ \\
\hline \multicolumn{8}{|l|}{ Indications } \\
\hline Herpes zoster & 34,919 & $\begin{array}{l}89.4 \\
(224.1)\end{array}$ & $\begin{array}{l}21(7- \\
56)\end{array}$ & $\begin{array}{l}6405 \\
(18.3)\end{array}$ & $\begin{array}{l}3700 \\
(10.6)\end{array}$ & $\begin{array}{l}2134 \\
(6.1)\end{array}$ & $\begin{array}{l}6405 \\
(18.3)\end{array}$ \\
\hline $\begin{array}{l}\text { Diabetic } \\
\text { peripheral } \\
\text { neuropathic } \\
\text { pain }\end{array}$ & 13,369 & $\begin{array}{l}221.9 \\
(330.1)\end{array}$ & $\begin{array}{l}71 \\
(28- \\
294)\end{array}$ & $\begin{array}{l}6044 \\
(45.2)\end{array}$ & $\begin{array}{l}4381 \\
(32.8)\end{array}$ & $\begin{array}{l}2894 \\
(21.6)\end{array}$ & $\begin{array}{l}6044 \\
(45.2)\end{array}$ \\
\hline Fibromyalgia & 21,051 & $\begin{array}{l}150.8 \\
(302.8)\end{array}$ & $\begin{array}{l}28 \\
(14- \\
117)\end{array}$ & $\begin{array}{l}6179 \\
(29.4)\end{array}$ & $\begin{array}{l}4026 \\
(19.1)\end{array}$ & $\begin{array}{l}2470 \\
(11.7)\end{array}$ & $\begin{array}{l}6179 \\
(29.4)\end{array}$ \\
\hline Epilepsy & 3,301 & $\begin{array}{l}515.5 \\
(603.5)\end{array}$ & $\begin{array}{l}220 \\
(35- \\
814)\end{array}$ & $\begin{array}{l}2085 \\
(63.2)\end{array}$ & $1748(53)$ & $\begin{array}{l}1400 \\
(42.4)\end{array}$ & $\begin{array}{l}2085 \\
(63.2)\end{array}$ \\
\hline $\begin{array}{l}\text { Other } \\
\text { musculoskeletal } \\
\text { diseases }\end{array}$ & 24227 & $\begin{array}{l}138.3 \\
(269.8)\end{array}$ & $\begin{array}{l}30 \\
(14- \\
113)\end{array}$ & $\begin{array}{l}7141 \\
(29.5)\end{array}$ & $\begin{array}{l}4499 \\
(18.6)\end{array}$ & $\begin{array}{l}2638 \\
(10.9)\end{array}$ & $\begin{array}{l}7141 \\
(29.5)\end{array}$ \\
\hline
\end{tabular}




\begin{tabular}{|c|c|c|c|c|c|c|c|}
\hline \multirow[b]{2}{*}{ Others } & \multirow[b]{2}{*}{17,570} & \multicolumn{2}{|c|}{$\begin{array}{l}\text { Duration of } \\
\text { persistence (days) }\end{array}$} & \multicolumn{4}{|c|}{ No. of patients with persistent pregabalin use } \\
\hline & & $\begin{array}{l}158.6 \\
(295.9)\end{array}$ & $\begin{array}{l}35 \\
(14- \\
141)\end{array}$ & $\begin{array}{l}5716 \\
(32.5)\end{array}$ & $\begin{array}{l}3847 \\
(21.9)\end{array}$ & $\begin{array}{l}2349 \\
(13.4)\end{array}$ & $\begin{array}{l}5716 \\
(32.5)\end{array}$ \\
\hline
\end{tabular}

Table 3

Proportion of prescribed maximum daily dose of pregabalin during follow-up

\begin{tabular}{|c|c|c|c|c|c|c|c|}
\hline \multirow[b]{2}{*}{ Indications } & \multirow[b]{2}{*}{$\begin{array}{l}\text { Mean } \\
\text { (SD) }\end{array}$} & \multicolumn{6}{|c|}{ Maximum dose prescribed } \\
\hline & & $<150 \mathrm{mg}$ & $\begin{array}{l}>=150 \mathrm{mg} \\
\sim \\
<300 \mathrm{mg}\end{array}$ & $\begin{array}{l}>=300 \mathrm{mg} \\
\sim \\
<450 \mathrm{mg}\end{array}$ & $\begin{array}{l}>=450 \mathrm{mg} \\
\sim \\
<600 \mathrm{mg}\end{array}$ & $>=600 \mathrm{mg}$ & Sum \\
\hline Herpes zoster & $\begin{array}{l}147.2 \\
(100.3)\end{array}$ & $\begin{array}{l}11257 \\
(32.2)\end{array}$ & $\begin{array}{l}21181 \\
(60.7)\end{array}$ & $\begin{array}{l}1986 \\
(5.7)\end{array}$ & $359(1)$ & $136(0.4)$ & 34919 \\
\hline $\begin{array}{l}\text { Diabetic peripheral } \\
\text { neuropathic pain }\end{array}$ & $\begin{array}{l}131.2 \\
(93)\end{array}$ & $\begin{array}{l}5900 \\
(44.1)\end{array}$ & $\begin{array}{l}6789 \\
(50.8)\end{array}$ & $580(4.3)$ & $76(0.6)$ & $24(0.2)$ & 13369 \\
\hline Fibromyalgia & $\begin{array}{l}139.2 \\
(89)\end{array}$ & $\begin{array}{l}8323 \\
(39.5)\end{array}$ & $\begin{array}{l}11332 \\
(53.8)\end{array}$ & $\begin{array}{l}1039 \\
(4.9)\end{array}$ & $294(1.4)$ & $63(0.3)$ & 21051 \\
\hline Epilepsy & $\begin{array}{l}200.9 \\
(145.4)\end{array}$ & $\begin{array}{l}763 \\
(23.1)\end{array}$ & $1717(52)$ & $\begin{array}{l}504 \\
(15.3)\end{array}$ & $247(7.5)$ & $70(2.1)$ & 3301 \\
\hline $\begin{array}{l}\text { Other } \\
\text { musculoskeletal } \\
\text { diseases }\end{array}$ & $\begin{array}{l}131.1 \\
(72.2)\end{array}$ & $\begin{array}{l}9825 \\
(40.6)\end{array}$ & $\begin{array}{l}13483 \\
(55.7)\end{array}$ & 749 (3.1) & $134(0.6)$ & $36(0.1)$ & 24227 \\
\hline Others & $\begin{array}{l}138.8 \\
(89.1)\end{array}$ & $\begin{array}{l}6799 \\
(38.7)\end{array}$ & $\begin{array}{l}9708 \\
(55.3)\end{array}$ & $823(4.7)$ & $184(1)$ & $56(0.3)$ & 6,433 \\
\hline Total & $\begin{array}{l}140.7 \\
(92.8)\end{array}$ & $\begin{array}{l}42867 \\
(37.5)\end{array}$ & $\begin{array}{l}64210 \\
(56.1)\end{array}$ & $\begin{array}{l}5681 \\
(5.0)\end{array}$ & $\begin{array}{l}1294 \\
(1.1)\end{array}$ & $385(0.3)$ & 114437 \\
\hline
\end{tabular}

Factors associated with persistent pregabalin use by one year.

The persistence rate at one year after the index date was $12.1 \%$. In multivariate logistic regression analysis, male gender $(\mathrm{OR}=1.14,95 \% \mathrm{Cl} 1.01-1.18, \mathrm{p}<0.001)$, older age $(\mathrm{OR}=1.01$ per year, $95 \% \mathrm{Cl}=1.01-1.01, \mathrm{p}<0.001)$, higher $\mathrm{CCl}$ score, and the initial concomitant prescription with gabapentin, duloxetine, tramadol, acetaminophen/tramadol, and lidocaine medicated plaster were associated with a significantly increased probability of persistent use of pregabalin at one year (Table 4). Compared with prescriptions from neurologists, those from rheumatologists $(\mathrm{OR}=1.64,95 \% \mathrm{Cl}=1.54-1.76, \mathrm{p}<0.001)$ and psychiatrists $(\mathrm{OR}=1.31,95 \% \mathrm{Cl}=$ $1.01-1.70, p=0.039$ ) were more likely to be persistent at one year. Among the different indications of pregabalin prescription, epilepsy $(\mathrm{OR}=8.04,95 \% \mathrm{Cl}=7.33-8.81, \mathrm{P}<0.001)$ was associated with the highest probability of continued use, followed by DPNP $(\mathrm{OR}=3.05,95 \% \mathrm{Cl}=2.85-3.27, \mathrm{p}<0.001)$, other musculoskeletal diseases (OR $=1.64,95 \% \mathrm{Cl}=1.54-1.75, \mathrm{p}<0.001)$, and fibromyalgia $(\mathrm{OR}=1.59,95 \% \mathrm{Cl}=1.48-1.70, \mathrm{p}<0.001)$, when compared to PHN. Besides, a higher initial pregabalin dose ( $\mathrm{OR}=1.12 \mathrm{per} 75 \mathrm{mg}, 95 \% \mathrm{Cl}=1.10-1.15, \mathrm{p}<0.001)$ was also associated with an increased probability of persistence at one year. On the other hand, concomitant 
prescription of anti-viral agents was associated with decreased likelihood of pregabalin use at one year (OR = $0.41,95 \% \mathrm{Cl}=0.35-0.47, \mathrm{p}<0.001)$ 
Table 4

Factor associated with persistent use of pregabalin at one year.

\begin{tabular}{|c|c|c|c|c|}
\hline \multirow[b]{2}{*}{ Variable } & \multicolumn{2}{|l|}{ Univariate } & \multicolumn{2}{|c|}{ Multivariable } \\
\hline & Odds Ratio $(95 \% \mathrm{Cl})$ & $\mathrm{p}_{\text {Value }}$ & $\begin{array}{l}\text { Odds } \\
\text { Ratio } \\
(95 \% \\
\mathrm{Cl})\end{array}$ & Value \\
\hline Male & $1.24(1.19-1.28)$ & $<.001$ & $\begin{array}{l}1.14 \\
(1.09- \\
1.18)\end{array}$ & $\begin{array}{l}< \\
0.001\end{array}$ \\
\hline Age, per year & $1(1-1)$ & 0.001 & $\begin{array}{l}1.01 \\
(1.01- \\
1.01)\end{array}$ & $\begin{array}{l}< \\
0.001\end{array}$ \\
\hline \multicolumn{5}{|l|}{ Monthly income NT\$ } \\
\hline NT 0-21,009 & 1 & & 1 & \\
\hline NT $210,10-36,825$ & $0.87(0.84-0.91)$ & $<_{0.001}$ & $\begin{array}{l}0.97 \\
(0.93- \\
1.01)\end{array}$ & 0.187 \\
\hline$>36,825$ & $0.75(0.71-0.79)$ & $\begin{array}{l}<.001 \\
0.01\end{array}$ & $\begin{array}{l}0.87 \\
(0.83- \\
0.92)\end{array}$ & $\begin{array}{l}< \\
0.001\end{array}$ \\
\hline \multicolumn{5}{|l|}{ Region of Taiwan } \\
\hline Northern region & 1 & & 1 & \\
\hline Central region & $0.94(0.9-0.98)$ & 0.007 & $\begin{array}{l}0.86 \\
(0.82- \\
0.9)\end{array}$ & $\begin{array}{l}<.001 \\
0.00\end{array}$ \\
\hline Southern region & $0.92(0.83-1.02)$ & 0.126 & $\begin{array}{l}0.79 \\
(0.71- \\
0.89)\end{array}$ & $\iota_{0.001}^{<}$ \\
\hline Eastern region & $0.9(0.86-0.94)$ & $\begin{array}{l}<.001 \\
0.00\end{array}$ & $\begin{array}{l}1 \\
(0.95- \\
1.05)\end{array}$ & 0.981 \\
\hline $\begin{array}{l}\text { Charlson Comorbidity } \\
\text { Index score, per } 1 \text { score }\end{array}$ & $1.06(1.05-1.06)$ & $\dot{0}_{0.001}$ & & \\
\hline \multicolumn{5}{|l|}{$\begin{array}{l}\text { Specialty of prescribing } \\
\text { physicians }\end{array}$} \\
\hline Neurology & 1 & & 1 & \\
\hline Anesthesiology & $0.99(0.91-1.07)$ & 0.715 & $\begin{array}{l}1.08 \\
(0.99- \\
1.17)\end{array}$ & 0.094 \\
\hline Physiatry & $0.73(0.65-0.82)$ & $\dot{0}_{0.001}$ & $\begin{array}{l}0.92 \\
(0.82- \\
1.03)\end{array}$ & 0.141 \\
\hline
\end{tabular}




\begin{tabular}{|c|c|c|c|c|}
\hline \multirow[b]{2}{*}{ Psychiatry } & \multicolumn{2}{|l|}{ Univariate } & \multicolumn{2}{|c|}{ Multivariable } \\
\hline & $1.28(1-1.64)$ & 0.047 & $\begin{array}{l}1.31 \\
(1.01- \\
1.7)\end{array}$ & 0.039 \\
\hline Rheumatology & $1.14(1.08-1.21)$ & <.001 & $\begin{array}{l}1.64 \\
(1.54- \\
1.76)\end{array}$ & $\dot{0.001}$ \\
\hline Others & $0.41(0.39-0.43)$ & $\begin{array}{l}< \\
0.001\end{array}$ & $\begin{array}{l}0.61 \\
(0.58- \\
0.64)\end{array}$ & $\dot{<} 001$ \\
\hline \multicolumn{5}{|l|}{ Indications } \\
\hline Herpes zoster & 1 & & 1 & \\
\hline $\begin{array}{l}\text { Diabetic peripheral } \\
\text { neuropathic pain }\end{array}$ & $4.24(4-4.51)$ & $\begin{array}{l}< \\
0.001\end{array}$ & $\begin{array}{l}3.05 \\
(2.85- \\
3.27)\end{array}$ & $\dot{<} 0.001$ \\
\hline Fibromyalgia & $2.04(1.92-2.17)$ & <.001 & $\begin{array}{l}1.59 \\
(1.48- \\
1.7)\end{array}$ & $\begin{array}{l}< \\
0.001\end{array}$ \\
\hline Epilepsy & $11.31(10.43-12.28)$ & $<.001$ & $\begin{array}{l}8.04 \\
(7.33- \\
8.81)\end{array}$ & $<.001$ \\
\hline $\begin{array}{l}\text { Other musculoskeletal } \\
\text { diseases }\end{array}$ & $1.88(1.77-1.99)$ & ¿. 001 & $\begin{array}{l}1.64 \\
(1.54- \\
1.75)\end{array}$ & $<.001$ \\
\hline Others & $2.37(2.23-2.52)$ & <.001 & $\begin{array}{l}1.98 \\
(1.85- \\
2.11)\end{array}$ & $\begin{array}{l}< \\
0.001\end{array}$ \\
\hline \multicolumn{5}{|c|}{ Concomitant medications } \\
\hline Gabapentin & $2.04(1.95-2.14)$ & $<.001$ & $\begin{array}{l}2.04 \\
(1.94- \\
2.15)\end{array}$ & $\dot{0} 001$ \\
\hline Duloxetine & $2.17(2.02-2.33)$ & $\begin{array}{l}< \\
0.001\end{array}$ & $\begin{array}{l}1.53 \\
(1.42- \\
1.65)\end{array}$ & $\stackrel{<}{0.001}$ \\
\hline NSAIDs & $0.56(0.54-0.58)$ & ¿. & $\begin{array}{l}0.72 \\
(0.7- \\
0.75)\end{array}$ & $<.001$ \\
\hline Acetaminophen & $0.61(0.59-0.64)$ & $<.001$ & $\begin{array}{l}0.79 \\
(0.76- \\
0.83)\end{array}$ & $\dot{0} 001$ \\
\hline Tramadol & $1.43(1.32-1.54)$ & $\begin{array}{l}< \\
0.001\end{array}$ & $\begin{array}{l}1.3 \\
(1.2- \\
1.42)\end{array}$ & $\stackrel{<}{<.001}$ \\
\hline
\end{tabular}




\begin{tabular}{|c|c|c|}
\hline & & Univariate \\
\hline Acetaminophen/tramadol & & $0.99(0.95-1.03)$ \\
\hline $\begin{array}{l}\text { Lidocaine medicated } \\
\text { plaster }\end{array}$ & & $1.2(1.01-1.42)$ \\
\hline Anti-viral agents & & $0.22(0.19-0.25)$ \\
\hline Daily dosage, per $75 \mathrm{mg}$ & & $1.16(1.13-1.19)$ \\
\hline \multicolumn{3}{|c|}{$\begin{array}{l}\text { Supplementary Table. The ICD diagnosis codes of indications for } \\
\text { pregabalin. }\end{array}$} \\
\hline & $\begin{array}{l}\text { ICD-9- } \\
\text { CM } \\
\text { codes }\end{array}$ & ICD-10 codes \\
\hline Herpes zoster & 053.x & B02.x, B00.9 \\
\hline $\begin{array}{l}\text { Diabetic peripheral } \\
\text { neuropathic pain }\end{array}$ & $250 . x$ & E08.x, E09.x, E10.x, E11.x, E13.x \\
\hline Fibromyalgia & 729.x & $\begin{array}{l}\text { M60.8x, M60.9, M79.0, M79.1, } \\
\text { M79.7, M79.9 }\end{array}$ \\
\hline Epilepsy & $\begin{array}{l}345 . x, \\
780.3 \\
780.39\end{array}$ & G40.x, R56.1, R56.9 \\
\hline $\begin{array}{l}\text { Other musculoskeletal } \\
\text { diseases }\end{array}$ & $\begin{array}{l}710 . x- \\
739 . x \\
\text { except } \\
729 . x\end{array}$ & $\begin{array}{l}\text { M05.x, M06.x, M12.x, M13.x, M15.x, } \\
\text { M17.x, M19.x, M35.x, M43.x, M45.x, } \\
\text { M46.x, M47.x, M48.x, M50.x, M79.x } \\
\text { (excluding M79.7) }\end{array}$ \\
\hline
\end{tabular}

\section{Discussion}

Pregabalin was most commonly prescribed for the treatment of pain in $\mathrm{PHN}(30.5 \%)$ and fibromyalgia $(18.4 \%)$ in Taiwan, and this was also reflected by a high proportion of concomitant prescription of other analgesics (Table 1). Most of the prescriptions were limited to short-term use, as evidenced by a mean duration of prescription lasting $149.5 \pm 299.8$ days (median 28 , interquartile range [IQR] 14-118), especially in PNH (89.4 \pm 224.1 days) and fibromyalgia ( $150.8 \pm 302.8$ days). Besides, the mean dose prescribed was $140.7 \pm 92.8 \mathrm{mg}$, which was lower than the doses used in clinical trials for $\mathrm{NeP}(3)$ and fibromyalgia (8), although the mean dose of prescriptions for epilepsy could be within the lower range of recommended doses (10).

The finding that pregabalin prescriptions were typically of short duration is in keeping with prior reports. According to a retrospective administrative claims data analysis from the United States, only $56.9 \%$ of patients initiating pregabalin for PHN obtained a refill during the one-year follow-up period (15). Similarly, $34 \%$ had just 
one single dispensed prescription for various indications, including epilepsy, generalized anxiety disorder, and neuropathic pain, indicating only $64 \%$ had further refills after the first prescription in Sweden (16). Besides, according to an Israeli study in fibromyalgia, the median time to discontinuation for antiepileptics, namely pregabalin and gabapentin, was 30 days (IQR 30-106 days) (17), and the mean prescription duration of pregabalin for pain was 52.2 days in a Japanese real-word study (18). In the present study, the persistence rates were $29.3 \%$ and $12.1 \%$ at 90 days and one year, respectively, indicating only a minority of patients continued to use pregabalin. All the above findings indicate that pregabalin prescriptions are usually discontinued early, which is a universal phenomenon.

A number of factors could be responsible for the short duration of pregabalin treatment for pain. As the NeP in PHN could resolve in months or years $(19,20)$, treatment would be no longer necessary after resolution of symptoms. More importantly, pregabalin could be prescribed off-label for the acute pain in herpes zoster rather than for PHN in some patients in the present cohort. In fact, $5.8 \%$ of pregabalin users were prescribed with antiviral agents at the same time (Table 1), and concomitant use of anti-viral agents was associated with decreased likelihood of pregabalin use at one year (OR $0.41,95 \% \mathrm{Cl} 0.35-0.47, \mathrm{p}<0.001)$ (Table 4). Although pregabalin is only indicated for $\mathrm{PNH}$, there is some evidence supporting its efficacy for acute pain of herpes zoster $(21,22)$. On the other hand, pregabalin could also be discontinued due to a lack of desired efficacy or the development of intolerable side effects. In fact, adverse effects of pregabalin treatment usually appear early in the treatment course, and usually resolve after prolonged use (11). Premature discontinuation preclude further escalation of the dosage, and thus could also account for the finding that most prescriptions were under-dosed, especially in pain disorders. Both could lead to poor treatment outcomes and hence compromised quality of life. An in-depth understanding of the dynamics of development of adverse events, would be helpful to optimize the prescription of pregabalin.

Among the different indications included, epilepsy patients had the greatest likelihood of long-term use, and PHN the least. Similar findings were reported in the Swedish study mentioned above (16), in which epilepsy and NeP had the best and worst persistence rates during follow-up. Generally speaking, studies evaluating drug treatment in epilepsy usually report high persistence or adherence rates (23-25). This could probably be attributed to the unremitting nature of most seizure disorders, as well as the devastating and potentially life-threating consequences of not receiving treatment. In contrast, in the American study mentioned above, the low persistence rate of pregabalin use in the treatment of $\mathrm{NeP}$ was, at least in part, attributed to suboptimal dosing, with $87 \%$ reaching $\geq 150 \mathrm{mg} /$ day and $27 \%$ reaching $\geq 300 \mathrm{mg} /$ day (15), which were higher than the corresponding figures of $62.5 \%$ and $6.4 \%$, respectively, in the present study (Table 3 ). Besides, the average doses prescribed for NeP (147.2 $\pm 100.3 \mathrm{mg}$ for PHN, $131.2 \pm 93 \mathrm{mg}$ for DPNP) (Table 3$)$ were lower than those in the American (187 mg for PHN) (15) and Swedish studies (275 mg for NeP) (16). The tolerability of pregabalin was reported to be comparable to that of placebo in clinical trials $(10,12-14)$. However, the majority of pivotal trials were carried out in North America and/or Europe. In fact, side effects, such as dizziness, somnolence, peripheral edema, and weight gain, were more common among Asians as compared to Caucasians (11). This could result from increased exposure to pregabalin due to the relatively lower average body weights among Asian patients, which could result in decreased tolerability, and possibly lower average doses prescribed.

The most important strength of the present study is the large-scale sample recruited, and the almost complete coverage of our $\mathrm{NHI}$ leads to the relative freedom from selection bias of the dataset. Besides, the data from NHIRD have been validated by a number of studies, and have yielded important findings that are widely accepted 
(26-28). On the other hand, the main weaknesses include the validity of the diagnosis coding and the lack of relevant clinical information regarding the initiation and discontinuation of the prescription. PHN or DPNP, and fibromyalgia could not be accurately coded in the ICD-9-CM coding system, and this remains an issue in the ICD10 coding system, except for fibromyalgia. Besides, it could be possible that fibromyalgia could be miscoded as other musculoskeletal diseases. However, when these diagnosis codes were associated with pregabalin prescriptions concomitantly, the probability that these codes correspond to the designated diagnoses should be high. Despite the above weaknesses, the present study could still provide some useful information regarding the pattern of pregabalin prescriptions in our country.

\section{Conclusion}

In conclusion, pain control in PHN and fibromyalgia is the most common indication for pregabalin use in Taiwan, although many of the prescriptions were limited to low dosage and short-term use. The exact reasons are unknown, and possibilities included off-label prescription for acute pain in herpes zoster, as well as an increased incidence of side effects in Asian populations. A thorough understanding of the temporal profile of adverse effects, as well as education for both the clinicians and patients, could help circumvent premature discontinuation and could therefore optimize the treatment outcome.

\section{Abbreviations}

CCl: Charlson Comorbidity Index; Cl: confidence interval; DPNP: diabetic peripheral neuropathic pain; ICD-9-CM: International Classification of Diseases, the Ninth Revision, Clinical Modification; IQR: interquartile range; OR: odds ratio; NeP: neuropahic pain; NHI: National Health Insurance; NHIRD: National Health Insurance Research Database; PHN: post-herpetic neuralgia; SD: standard deviation

\section{Declarations}

\section{Ethics approval}

The study protocols were approved by the Institutional Review Board of National Yang-Ming University (approval No. YM105009E).

\section{Consent for publication}

Not applicable

\section{Availability of data and material}

The NHIRD is publicly available from the Taiwan NHI.

\section{Competing interests}

YFW has received honoraria as a speaker from Taiwan branches of Allergan, Pfizer, Eli Lilly, Novartis, and Sanofi. He has received research grants from the Taiwan Minister of Technology and Science, and Taipei Veterans General Hospital. 
SJW has served on the advisory boards of Eli Lilly, Daiichi-Sankyo, and Taiwan Novartis. He has received honoraria as a moderator from Allergan, Pfizer, Eli Lilly, Bayer, and Eisai. He has received research grants from the Taiwan Minister of Technology and Science, National Yang-Ming University, Taipei Veterans General Hospital, Taiwan Headache Society, Taiwan Pfizer, Taiwan Novartis, and Taiwan Eli Lilly. CWT, YCY, YCC, and BCS declared no conflict of interest.

\section{Funding}

This study was sponsored by Pfizer, Taiwan.

\section{Authors' contributions}

YFW and YTC: drafting initial manuscript, manuscript revision, study design, data acquisition, data analysis; CWT, YCY, YCC, BCS: data acquisition, data analysis; SJW: study design, manuscript revision. All authors approved the final manuscript.

\section{Acknowledgements}

We acknowledge supports from Taipei Veterans General Hospital, National Yang-Ming University, and Taipei Medical University.

\section{References}

1. Calandre EP, Rico-Villademoros F, Slim M. Alpha2delta ligands, gabapentin, pregabalin and mirogabalin: a review of their clinical pharmacology and therapeutic use. Expert Rev Neurother. 2016;16(11):1263-77.

2. Dworkin RH, O'Connor AB, Backonja M, Farrar JT, Finnerup NB, Jensen TS, et al. Pharmacologic management of neuropathic pain: evidence-based recommendations. Pain. 2007;132(3):237-51.

3. Finnerup NB, Attal N, Haroutounian S, McNicol E, Baron R, Dworkin RH, et al. Pharmacotherapy for neuropathic pain in adults: a systematic review and meta-analysis. Lancet Neurol. 2015;14(2):162-73.

4. Dworkin RH, O'Connor AB, Audette J, Baron R, Gourlay GK, Haanpaa ML, et al. Recommendations for the pharmacological management of neuropathic pain: an overview and literature update. Mayo Clin Proc. 2010;85(3 Suppl):S3-14.

5. Bril V, England J, Franklin GM, Backonja M, Cohen J, Del Toro D, et al. Evidence-based guideline: Treatment of painful diabetic neuropathy: report of the American Academy of Neurology, the American Association of Neuromuscular and Electrodiagnostic Medicine, and the American Academy of Physical Medicine and Rehabilitation. Neurology. 2011;76(20):1758-65.

6. Bril V, England JD, Franklin GM, Backonja M, Cohen JA, Del Toro DR, et al. Evidence-based guideline: treatment of painful diabetic neuropathy--report of the American Association of Neuromuscular and Electrodiagnostic Medicine, the American Academy of Neurology, and the American Academy of Physical Medicine \& Rehabilitation. Muscle Nerve. 2011;43(6):910-7.

7. Calandre EP, Rico-Villademoros F, Slim M. An update on pharmacotherapy for the treatment of fibromyalgia. Expert Opin Pharmacother. 2015;16(9):1347-68.

8. Macfarlane GJ, Kronisch C, Dean LE, Atzeni F, Hauser W, Fluss E, et al. EULAR revised recommendations for the management of fibromyalgia. Ann Rheum Dis. 2017;76(2):318-28. 
9. Arnold LM, Clauw DJ. Challenges of implementing fibromyalgia treatment guidelines in current clinical practice. Postgrad Med. 2017;129(7):709-14.

10. Kanner AM, Ashman E, Gloss D, Harden C, Bourgeois B, Bautista JF, et al. Practice guideline update summary: Efficacy and tolerability of the new antiepileptic drugs II: Treatment-resistant epilepsy: Report of the Guideline Development, Dissemination, and Implementation Subcommittee of the American Academy of Neurology and the American Epilepsy Society. Neurology. 2018;91(2):82-90.

11. Ogawa S, Satoh J, Arakawa A, Yoshiyama T, Suzuki M. Pregabalin treatment for peripheral neuropathic pain: a review of safety data from randomized controlled trials conducted in Japan and in the west. Drug Saf. 2012;35(10):793-806.

12. Waldfogel JM, Nesbit SA, Dy SM, Sharma R, Zhang A, Wilson LM, et al. Pharmacotherapy for diabetic peripheral neuropathy pain and quality of life: A systematic review. Neurology. 2017;88(20):1958-67.

13. Straube S, Derry S, Moore RA, McQuay HJ. Pregabalin in fibromyalgia: meta-analysis of efficacy and safety from company clinical trial reports. Rheumatology (Oxford). 2010;49(4):706-15.

14. Hauser W, Bernardy K, Uceyler N, Sommer C. Treatment of fibromyalgia syndrome with gabapentin and pregabalin--a meta-analysis of randomized controlled trials. Pain. 2009;145(1-2):69-81.

15. Johnson P, Becker L, Halpern R, Sweeney M. Real-world treatment of post-herpetic neuralgia with gabapentin or pregabalin. Clin Drug Investig. 2013;33(1):35-44.

16. Wettermark B, Brandt L, Kieler H, Boden R. Pregabalin is increasingly prescribed for neuropathic pain, generalised anxiety disorder and epilepsy but many patients discontinue treatment. Int J Clin Pract. 2014;68(1):104-10.

17. Ben-Ami Shor D, Weitzman D, Dahan S, Gendelman O, Bar-On Y, Amital D, et al. Adherence and Persistence with Drug Therapy among Fibromyalgia Patients: Data from a Large Health Maintenance Organization. J Rheumatol. 2017;44(10):1499-506.

18. Ushida T, Matsui D, Inoue T, Yokoyama M, Takatsuna H, Matsumoto T, et al. Recent prescription status of oral analgesics in Japan in real-world clinical settings: retrospective study using a large-scale prescription database. Expert Opin Pharmacother. 2019;20(16):2041-52.

19. Bouhassira D, Chassany O, Gaillat J, Hanslik T, Launay O, Mann C, et al. Patient perspective on herpes zoster and its complications: an observational prospective study in patients aged over 50 years in general practice. Pain. 2012;153(2):342-9.

20. McKendrick MW, Ogan P, Care CC. A 9 year follow up of post herpetic neuralgia and predisposing factors in elderly patients following herpes zoster. J Infect. 2009;59(6):416-20.

21. Jensen-Dahm C, Rowbotham MC, Reda H, Petersen KL. Effect of a single dose of pregabalin on herpes zoster pain. Trials. 2011;12:55.

22. Kanodia SK, Singhal KC. A study on efficacy of Pregabalin in acute Herpetic Neuralgia. Ann Neurosci. 2011;18(4):148-50.

23. Faught E, Helmers S, Thurman D, Kim H, Kalilani L. Patient characteristics and treatment patterns in patients with newly diagnosed epilepsy: A US database analysis. Epilepsy Behav. 2018;85:37-44.

24. Aylward BS, Rausch JR, Modi AC. An examination of 1-year adherence and persistence rates to antiepileptic medication in children with newly diagnosed epilepsy. J Pediatr Psychol. 2015;40(1):66-74. 
25. Lee YK, Ah YM, Choi YJ, Cho YS, Kim KJ, Lee JY. Antiepileptic drug adherence and persistence in children with epilepsy attending a large tertiary care children's hospital. Epileptic Disord. 2016;18(4):408-17.

26. Shih CJ, Chu H, Chao PW, Lee YJ, Kuo SC, Li SY, et al. Long-term clinical outcome of major adverse cardiac events in survivors of infective endocarditis: a nationwide population-based study. Circulation. 2014;130(19):1684-91.

27. Wang YF, Chen YT, Luo JC, Chen TJ, Wu JC, Wang SJ. Proton-Pump Inhibitor Use and the Risk of First-Time Ischemic Stroke in the General Population: A Nationwide Population-Based Study. Am J Gastroenterol. 2017;112(7):1084-93.

28. Peng KP, Chen YT, Fuh JL, Tang CH, Wang SJ. Migraine and incidence of ischemic stroke: A nationwide population-based study. Cephalalgia. 2017;37(4):327-35. 